\title{
Predictive and prognostic impact of tumour-infiltrating lymphocytes in triple-negative breast cancer treated with neoadjuvant chemotherapy
}

\author{
Carmen Herrero-Vicent', Angel Guerrero', Joaquin Gavilá1, Francisco Gozalbo², Abraham Hernández¹, Sergio Sandiego', \\ Maria Asunción Algarra ${ }^{1}$, Ana Calatrava², Vicente Guillem-Porta1 and Amparo Ruiz-Simón ${ }^{1}$ \\ ${ }^{1}$ Medical Oncology Department, Valencian Institute of Oncology, 46008 Valencia, Spain \\ ${ }^{2}$ Pathology Department, Valencian Institute of Oncology, 46008 Valencia, Spain
}

Correspondence to: C Herrero-Vicent. Email: carmendcn@hotmail.com

\begin{abstract}
Introduction: In locally and locally advanced triple-negative breast cancer (TNBC), neoadjuvant chemotherapy (NAC) only induces a pCR in 30-35\% of patients. Clinical and pathological factors are not enough to distinguish the patients who have no chance of a pCR or not. The tumour microenvironment is critical for cancer and tumour-infiltrating lymphocytes (TIL). Moreover, the NAC scenario is the perfect setting to study possible changes in TIL levels.
\end{abstract}

Material and methods: Using our prospective maintained breast cancer (BC) database, we identified 164 TNBC patients treated with NAC between 1998 and 2015 with enough samples of diagnostic biopsy and after surgery. Evaluation of TILs before and after NAC followed a standardised methodology for visual assessment on haematoxylin-eosin sections and the amounts of TILs were quantitated in deciles. We categorised lymphocyte-predominant breast cancer cutoff according to a receiver operating characteristic (ROC) analysis. We categorised LPBC as involving $>40 \%$ lymphocytic infiltration tumour stroma. The primary end point was predictive value of TILs to NAC, and the secondary end point was disease-free survival (DFS). DFS was analysed using the Kaplan-Meier method and the groups were compared with a long-rank test. Univariate and multivariate Cox models were used to generate hazard ratios for determining associations between variables such as TIL after NAC and DFS.

Results: A total of 164 TNBC patients were treated with NAC and surgery. The main patients' characteristics are listed in Table 1. We identify different pathological complete response to anthracycline and taxane-based NAC; LPBC subgroup 51 from 58 patients (88\%) pCR versus non- lymphocyte-predominant breast cancer (LPBC) subgroup 10 from 106 (9\%) pCR, $p=0.001$. At a median follow-up of 78 months, LPBC was associated with better DFS; the three-year Kaplan-Meier estimates for DFS were $2 \%$ and $30 \%$ for patients with LPBC and non-LPBC, respectively, $p=0.01$. Univariate and multivariate analysis confirmed TIL to be an independent prognostic marker of DFS.

Published: 15/08/2017

Received:15/02/2017

ecancer 2017, 11:759 https://doi.org/10.3332/ecancer.2017.759

Copyright: (C) the authors; licensee ecancermedicalscience. This is an Open Access article distributed under the terms of the Creative Commons Attribution License (http://creativecommons.org/licenses/by/3.0), which permits unrestricted use, distribution, and reproduction in any medium, provided the original work is properly cited. 
Conclusions: Tumour-infiltrating lymphocytes could be routinely used in locally advanced TNBC treated with anthracycline and taxane, such as biomarker, to be enabled the identification of different two subgroups: LPBC patients have a very high response to NAC pCR $88 \%$, meanwhile non-LPBC patients only achieve $9 \%$. Moreover, non-LPBC patients have a worse prognosis than LPBC patients. This data verified the predictive and prognostic value of TIL.

Keywords: triple-negative breast cancer, tumour-infiltrating lymphocytes, predictive, prognostic value

\section{Introduction}

Triple-negative breast cancer (TNBC) is a subtype of breast cancer with a really bad prognosis. In locally advanced TNBC treated with neoadjuvant chemotherapy (NAC) only $30-35 \%$ of patients with TNBC get pCR, defined as no residual invasive carcinoma in breast (ypT0 or ypTis) and negative lymph node status (ypN0) [1]. Nowadays, NAC therapy is based on the patient's characteristics, tumour size, nodes, oestrogen and progesterone receptors, overexpression of human epithelial growth factor receptor, grade and ki67 [2]. However, these factors are not enough to distinguish the patients who have no chance of a pCR or not [1].

Recent studies suggest that the tumour microenvironment is critical for survival, invasive growth, and metastasis of the cancer [3]. Indeed, the host immune system responds to fight cancer; normal breast tissue does not contain aggregates of immune cells, but breast tumours and stroma display higher levels of immune cells infiltrates [4]. The median percentage of stromal tissue infiltrated with tumour-infiltrating lymphocytes (TIL) in TNBC was $20 \%$ in TNBC [5].

TNBC treated with NAC is the perfect setting to study possible changes in TIL levels, since we have a histological sample pre-treatment after NAC. Basal TILs or pre-NAC could act as such a predictive marker for response to standard NAC [6-8]. Furthermore, in patients without pCR, TILs in residual carcinoma could help in assessing risk. After all, new strategies should be considered to improve the outcome of TNBC patients $[1,9]$.

Criteria to evaluate TILs are different depending on the study. Cut-offs to divide between lymphocytic-predominant breast cancer (stromal TILs > 50\%) were used in some studies [6-8], while others used different cut-offs [5, 8, 10]. In 2015, an international TIL breast cancer working group recommended a scoring system for TILs involving haematoxylin-eosin (HE) staining for routine practice [11]. We aimed to investigate the utility of TILs using this scoring approach.

\section{Materials and methods}

\section{Patients}

A retrospective, single-institution study was conducted at the Valencian Institute of Oncology with ethics committee approval. The study is based on a database maintained for patients with locally advanced breast cancer who received neoadjuvant treatment between 1998 and 2015.

Patients were included in the study if they had TNBC [oestrogen receptor-, progesterone receptor-, and human epidermal growth factor receptor type 2- (ER- PR- and HER2-)] The threshold for ER and PR positivity was set at $1 \%$ using immunochemical staining. HER2 was positive if tumour cells showed $3+$ by $\mathrm{IHC}$ or $2+$ by $\mathrm{IHC}$ with amplification using silver in situ hybridisation. Patients were treated with neoadjuvant chemotherapy (NAC) based on anthracycline and taxane regimen.

Clinicopathological information was obtained from the database: age, histological classification, grade, tumour size, lymph node status, ki67, lymphovascular invasion, response to NAC and adjuvant treatment.

\section{Samples}

Core biopsies before NAC and surgical specimens obtained after NAC were reviewed. The largest size of tumours, histologic type and grade, lymphovascular invasion, proportion of ductal carcinoma in situ (DCIS), number of positive lymph nodes, and treatment response in breast and lymph nodes were evaluated. Tumour size and extent in breast and lymph nodes were assessed according to 
the recommendation proposed by Provenzano and the histologic type and grade were defined in accordance with the World Health Organisation classification and classified using the modified Scarff-Bloom-Richardson grading system, respectively.

HE samples were reviewed by a breast pathologist (FG) who was blinded to the patient profiles. He defined TIL score as the proportion of the stromal area infiltrated by lymphocytes following the recommendations of the International TIL Breast Cancer Working Group [7].

Ninety-six per cent of stored HE samples were retrieved for TIL assessment. A representative slide containing a relatively high amount of lymphocytic infiltration around invasive cancer was selected for each patient. The evaluation of TILs was following a standardised methodology for visual assessment on HE sections and the amounts of TILs were quantitated in deciles. Due to the heterogeneity of TILs, with different intensities of lymphocytes in different areas, hot spots at the invasive edge were avoided.

We define 'CTIL' as TIL in core biopsies before NAC and from patients without pathological complete response 'ypTIL' as TIL in surgical specimens obtained after NAC.

\section{Outcomes}

Predictive information was obtained from electronic charts. Pathological complete response (pCR) was defined as no residual invasive carcinoma in breast (ypT0 or ypTis) and negative lymph node status (ypNO).

Prognostic information was retrospectively obtained from a maintained clinical database. Disease free survival (DFS) was defined as the period of time between surgery and breast cancer relapsed, death of any cause or latest follow-up.

\section{Statistical analysis}

Data were analysed using SPSS version 20.0.

We categorised lymphocyte-predominant breast cancer (LPBC) cut-off according to a COR analysis.

The association between clinical and pathological parameters was tested with $\mathrm{X}^{2}$ test for categorical variables. Mean differences were studied with the $t$-test.

To identify variables associated with an increased recurrence, we performed firstly Kaplan-Meier curves. All events were measured from the date of histological diagnosis. The statistical significance between survival curves was determined by a log-rank test between two groups. Then, significant variables were included in a multivariate analysis using Cox proportional hazards. The median follow-up period for surviving patients was 78 months. All tests were two tailed, and $p<0.05$ was significant.

\section{Results}

\section{Patients' characteristics}

A total of 756 patients diagnosed with locally advanced invasive carcinomas and treated with NAC followed by surgery were identified from 1998 to 2015.

Based on the criteria described, 181 patients were diagnosed with TNBC, and of these, 164 patients (90.6\%) had enough samples available before and after NAC.

The median age was 49 years (range 29-81). The main clinical and pathological patients' characteristics are described in Table 1.

Fifty-nine per cent were treated with adriamicyn $60 \mathrm{mg} / \mathrm{m}^{2}$ - ciclofosfamide $600 \mathrm{mg} / \mathrm{m}^{2} / 21$ days $\times 4$ cycles followed by weekly taxane $\times 12$ cycles. Twenty per cent of patients received three-weekly docetaxel. Three per cent of patients were not treated with anthracycline and $5 \%$ with taxane-based NAC. Fifty per cent were treated with breast conservatory surgery (BCS), but $5.4 \%$ re-resections were needed to get $99 \%$ negative margins; $37.2 \%$ of patients presented pCR (ypT0/ypTis ypN0); $88 \%$ received adjuvant radiotherapy. The NAC regimen and surgery approach are also detailed in Table 1. 
Table 1. Baseline patient and tumour characteristics.

\begin{tabular}{|c|c|}
\hline Characteristics & $\%$ de patients \\
\hline Premenopausial & 57 \\
\hline \multicolumn{2}{|l|}{ Clinical TNM } \\
\hline $4 \| \mathrm{A}$ & 31 \\
\hline$+\mathrm{IIB}$ & 32 \\
\hline$+\quad I I A$ & 15 \\
\hline$+1 I I B$ & 10 \\
\hline$+111 \mathrm{C}$ & 12 \\
\hline \multicolumn{2}{|l|}{ Histology } \\
\hline$\$$ Ductal & 67 \\
\hline \& Lobulillar & 33 \\
\hline Grade 3 & 67 \\
\hline Linfovascular invasion & 9 \\
\hline Positive lymph node with biopsy prior NAC & 61 \\
\hline \multicolumn{2}{|l|}{ Neoadjuvant chemotherapy } \\
\hline$+\mathrm{AC}+$ Paclitaxel & 59 \\
\hline \&AC+Docetaxel & 20 \\
\hline+ Others & 21 \\
\hline$\$$ No taxanes & 5 \\
\hline * No anthracyclines & 3 \\
\hline Breast conservative surgery & 50 \\
\hline Re-resection & 5.4 \\
\hline Negative margins & 99 \\
\hline pCR( урTO/is yрNO) & 37.2 \\
\hline \multicolumn{2}{|l|}{ Residual tumor } \\
\hline $4 \mathrm{IA}$ & 24.1 \\
\hline$+\| \mathrm{A}$ & 15.2 \\
\hline$+\| \mathrm{IB}$ & 7.3 \\
\hline$+\| I I A$ & 12.1 \\
\hline$+\mathrm{IIIB}$ & 0.6 \\
\hline$+1 I I C$ & 4.8 \\
\hline Adjuvant radiotherapy & 88.4 \\
\hline
\end{tabular}

Table 2. Detailed distributions of clinical and pathological characteristics between LPBC group and non-LPBC group.

\begin{tabular}{|l|l|l|l|}
\hline \multicolumn{1}{|c|}{ Variable } & \multicolumn{2}{c|}{ TILs level } & \multicolumn{1}{c|}{$\mathrm{X}^{2}$} \\
\hline & HIGH & LOW & \\
\hline Age < 40 y & $32(55 \%)$ & $60(56 \%)$ & p.345 \\
\hline Stage TNM & & & \\
\hline Ila & $22(38 \%)$ & $29(28 \%)$ & p.205 \\
\hline Ilb & $22(38 \%)$ & $32(30 \%)$ & \\
\hline IIla & $7(12 \%)$ & $18(17 \%)$ & \\
\hline IIIb & $3(5 \%)$ & $10(9 \%)$ & \\
\hline IIIc & $4(7 \%)$ & $17(16 \%)$ & \\
\hline Histology & & & \\
\hline Ductal & $33(57 \%)$ & $64(60 \%)$ & p.060 \\
\hline Lobular & $25(43 \%)$ & $42(40 \%)$ & \\
\hline Grade 3 & $37(64 \%)$ & $32(30 \%)$ & p.005 \\
\hline DCIS & $6(10 \%)$ & $7(6 \%)$ & p.486 \\
\hline LVI & $6(10 \%)$ & $12(11 \%)$ & p.106 \\
\hline Ki67>50 & $50(86 \%)$ & $90(85 \%)$ & p.270 \\
\hline NAC & & & \\
\hline Anthracycline+taxane & $47(81 \%)$ & $98(92 \%)$ & p.570 \\
\hline pCR (ypT0/is, ypN0) & $51(88 \%)$ & $10(9 \%)$ & p.001 \\
\hline ResidualTumor & & & \\
\hline Multifocal & $4(7 \%)$ & $24(22 \%)$ & p.001 \\
\hline Conservative surgery & $39(67 \%)$ & $43(41 \%)$ & p.001 \\
\hline Adjuvant Radiotherapy & $51(88 \%)$ & $93(88 \%)$ & p.754 \\
\hline Quimioterapia adyuvante & $12(21 \%)$ & $47(44 \%)$ & p.078 \\
\hline
\end{tabular}




\section{TILs analysis}

The detailed distribution of basal TILs (before NAC) is detailed in Table 2. The main samples (57\%) presented basal TILs from $1 \%$ to $10 \%$. The lymphocyte-predominant breast cancer (LPBC) cut-off was 40\% ( $p=0.001)$. We categorised LPBC as involving > 40\% lymphocytic infiltration tumour stroma.

Fifty-eight patients (35.4\%) were LPBC, meanwhile 106 patients $(64.6 \%)$ were non-LPBC.

The detailed distributions of clinical and pathological characteristics are summarised in Table 2. There were no differences between median age, histology, clinical stage, lymphovascular invasion, ki67 marker, neither NAC regimen. However, LPBC group presented higher histological grade $3(64 \%$ vs. $34 \% p=0.005)$, higher BCS (67 \% vs. $40 \%, p=0.001)$ than non-LPBC.

\section{Predictive value of TIL}

Fifty-one of 58 (87\%) patients from LPBC group and 10 from 106 (9.4\%) from non-LPBC group achieved a pCR (Table 2). LPBC group presented higher pCR than non-LPBC ( $87 \%$ vs. $9 \% p=0.001)$. Breast tumour pCR (ypT0/is) and lymph nodes pCR (ypN0) were also higher in LPBC tan non-LPBC ( $86 \%$ vs. $12.2 \% p=.001)$ and $(93 \%$ vs. $45 \% p=0.001)$, respectively.

\section{Prognostic value of TIL}

At a median follow-up of 78 months, 41 recurrences (25\%) were observed after standard NAC and curative surgery; 36 recurrences $(33.9 \%)$ in the non-LPBC group and five recurrences $(8.5 \%)$ in LPBC $(p=0.005)$.

Figure 1 illustrates DFS curves according to LPBC and non-LPBC groups. TILs (high and low levels) proved to have significant prognostic value $(p=0.005)$ regarding DFS. Median DFS in non-LPBC was 20 months (IC 95\% 10-29 months) and LPBC was 97 months (IC $95 \%$ 27-166 months). At 3 years, LPBC presented $2 \%$ of recurrences meanwhile non-LPBC presented $30 \%(p=0.01)$.

By univariate analysis, the variables associated with an increased recurrence were clinical stage (cTNM), pCR (ypTO/is ypN0), TILs levels before NAC (cTNM), and after NAC-TILs levels (ypTILs).

By multivariate analysis, the variables associated with an increased recurrence were RCP $(\mathrm{HR} 10, p=0.014)$ y ypTIL $(\mathrm{HR} 0.8, p=0.016)$ Univariate and multivariate analysis details are described in Tables 3 and 4.

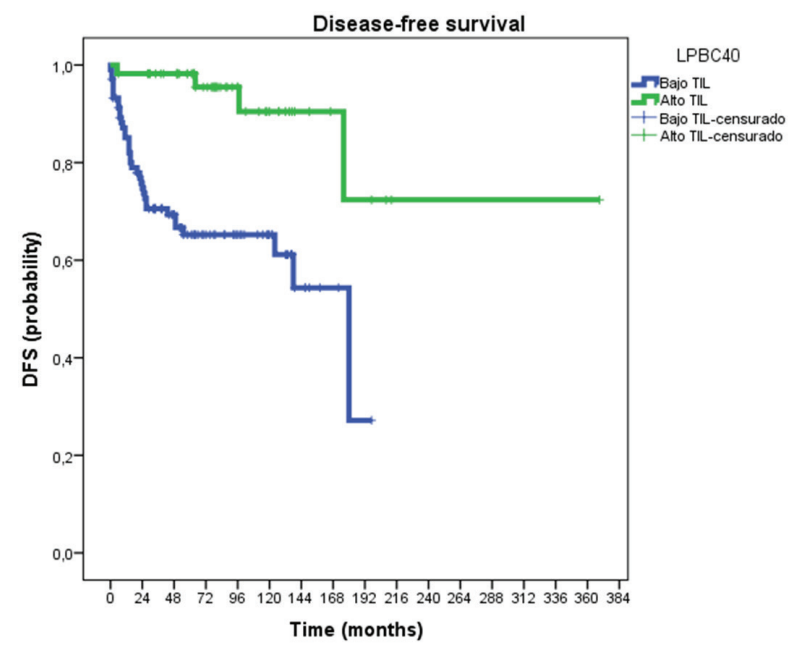

Figure 1. Disease-free survival curves according to LPBC and non-LPBC groups. 
Table 3. Univariate analysis details: variables associated with an increased recurrence.

\begin{tabular}{|c|c|c|}
\hline Variable & Recurrence at 6y & P (log-rank test) \\
\hline \multicolumn{3}{|l|}{ cTNM } \\
\hline $4 \mathrm{Ila}$ & 3 & p.002 \\
\hline$\$ \quad l l b$ & 6 & \\
\hline$+\quad 111 \mathrm{a}$ & 4 & \\
\hline$+111 \mathrm{lb}$ & 6 & \\
\hline$+\mathrm{IIIC}$ & 7 & \\
\hline \multicolumn{3}{|l|}{ cTIL } \\
\hline$+<40 \%$ & 22 & p.001 \\
\hline$+>40 \%$ & 9 & \\
\hline \multicolumn{3}{|l|}{$\mathrm{RCp}$ (ypTO/is ypNO) } \\
\hline$\$$ Yes & 4 & p.001 \\
\hline$+\mathrm{No}$ & 20 & \\
\hline \multicolumn{3}{|l|}{ ypTIL } \\
\hline$+1 \%$ & 18 & p.002 \\
\hline $4>1 \%$ & 2 & \\
\hline
\end{tabular}

CTIL $=$ TIL in core biopsies before NAC.

ypTIL = TIL in surgical specimens obtained after NAC.

$\mathrm{RCp}=$ Pathological complete response.

Table 4. Multivariate analysis details: variables associated with an increased recurrence.

\begin{tabular}{|c|r|r|}
\hline Variable & HR & P (log -rank test) \\
\hline RCp (ypT0/is ypN0) & & \\
+ Yes & (Reference ) 1 & p.030 \\
\hline+ No & 9.01 & \\
\hline ypTIL & & \\
\hline$+1 \%$ & (Reference ) 1 & p.016 \\
\hline$>1 \%$ & 0.81 & \\
\hline
\end{tabular}

ypTIL $=$ TIL in surgical specimens obtained after NAC.

$\mathrm{RC} p=$ Pathological complete response.

\section{Discussion}

In summary, we investigate the utility of TILs following the scoring system of an international TIL breast cancer working group [12]. In this retrospective study, we report the predictive and prognostic value of TILs in locally advanced TNBC treated with NAC based on anthracyclines and taxanes.

\section{Cut-off point}

We recognised the heterogeneity of TILs cut-off in order to divide between LPBC and non-LPBC [5, 10, 11]. TILs were analysed as a continuous variable, and also as a dichotomous variable with a cut-off. Some studies defined LPBC (stromal TILs > 50\%) [5, 8], while others used different cut-offs [10, 11]. For example, Hida et al [3] divided them into three different groups: the high-TIL (stromal and/or intratumoural TIL > 60\%) intermediate-TIL (TIL from 10\% to 60\%) and low-TIL (TIL < 10\%) groups were different. However, in Hida's study, the difference between these three groups was not significant because of the absence of events in the high-TIL group.

In our study, we performed a ROC curve and we categorised LPBC as involving $>40 \%$ lymphocytic infiltration tumour stroma. However, the diversity and richness of the antitumour immune response is, of course, severely underestimated by assessment with H\&E.9 


\section{Predictive value}

There are data which support that basal TIL or before NAC could be a predictive value in locally advanced TNBC treated with NAC based on anthracyclines $[5,9,17]$.

Denkert et al [11] demonstrated this in a retrospective study of 1058 patients from GEPADUO and GEPARTRIO trials with breast cancer treated with neoadjuvant cancer with anthracyclines and taxanes. Patients with TIL levels higher than $60 \%$, had higher rates of pCR compared to patients with low levels of TIL diagnosis (40\% vs. $7.2 \%)$. Another study [3], in which 474 patients were included with locally advanced TNBC and Her2 treated with NAC, suggested that TNBC with high levels of TIL at diagnosis had higher pCR than patients with low levels of TIL (37\% vs. $16 \%)$.

In the GEPARSIXTO study [15], patients with LPBC who were treated with carboplatin in addition to anthracycline and taxane had impressive pCR rates: $74 \%$ versus $43 \%$ compared with the $\mathrm{pCR}$ rate of patients with LPBC treated without carboplatin $(p=0.005)$.

In our study, we identify different pCR to anthracycline and taxane-based NAC: LPBC subgroup $88 \%$ pCR versus non-LPBC subgroup $9 \%$ pCR, $p=0.001$ (Figure 2).

TIL determination could be considered as a diagnostic in locally advanced TNBC patients treated with NAC based on anthracycline and taxanes because it represents a surrogate marker for efficacy [9].

Chemotherapy induces changes in the immune response, which are partially characterised, although some studies suggest that there could be cooperation between both of them [18-21]. In our study, the correlation between the two was evaluated, the targeted tumour belonging to the non-LPBC group did not change after receiving NAC.

\section{Prognostic value}

TILs have been evaluated in the past 5 years in nearly 16.000 patients in prospective studies with clinical follow-up data available, which highlights a rapid accumulation of evidence [5, 7, 10, 17, 22]. The BIG-02-98 trial, with 2009 breast cancer patients and 256 with TNBC, suggested that higher TIL levels were a significant favourable prognostic factor because they were associated to higher DFS and OS [5].

Two different studies ECOG 2197 and 1199 with 481 TNBC patients confirmed that higher TIL levels were a favourable prognostic factor [7].

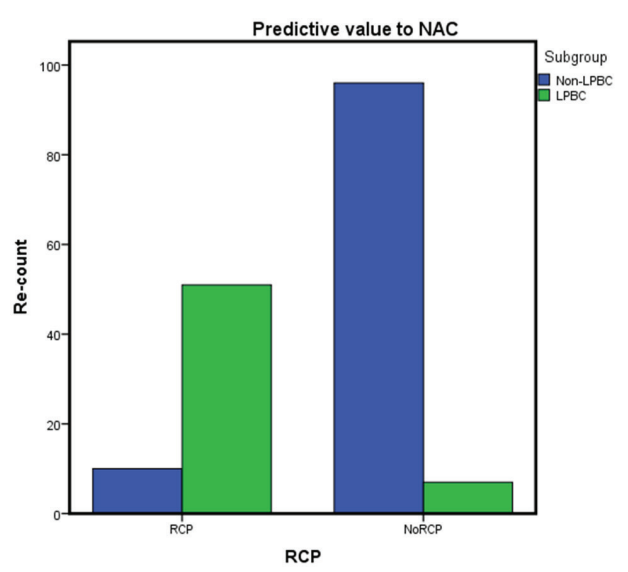

Figure 2. Pathological complete response to anthracycline and taxane-based NAC. 
Dieci et al reported the prognostic value of TILs in residual disease after NAC in TNBC [8]. Their survival curves for the high-TIL (stromal and/or intratumoural TIL > 60\%) intermediate-TIL (TIL from 10\% to 60\%) and low-TIL (TIL < 10\%) groups were different. However, in Hida's study, the difference between these three groups was not significant because of the absence of events in the high-TIL group [3].

Therefore, Simon et al [23] reported that the prognostic value of TILs in TNBC could be considered as Level-I evidence.

In our study, at a median follow-up of 78 months, LPBC was associated with better DFS; the three-year Kaplan-Meier estimates for DFS were $2 \%$ and $30 \%$ for patients with LPBC and non-LPBC, respectively, $p=0.01$. Moreover, univariate and multivariate analysis confirmed TIL to be an independent prognostic marker of DFS.

Although our findings may not change standard NAC options for locally advanced TNBC, TIL evaluation could be included in the standard histopathological practise as a prognostic factor.

\section{TILs in residual disease}

Patients with residual disease following NAC in TNBC are considered a poor prognosis group [24]. The standard of care for patients with TNBC who have residual disease after NAC is observation. However, new effective therapies in reducing recurrences are unknown.

The combination of TIL after NAC and pCR could enable patient stratification in different groups. The presence of TILs in residual disease could stratify patients and confers a better prognosis in non-pCR with LPBC patients. However, tumour-intrinsic signalling pathways related to immunity remains poorly understood [25-27].

Patients with LPBC

- And pCR have an excellent outcome

- Patients who do not achieve pCR have a good outcome but might require further enhancement of immunity by targeting counterregulatory mechanism that prevent tumour elimination.

Patients with non-LPBC

- And pCR also have a good outcome but might require therapies aimed at establishing immune infiltration by tumour-specific $T$ cells, which could be achieved through creating a more permissive immune microenvironment.

- Patients who do not achieve pCR have worse outcomes and might benefit from more toxic approaches. In this subgroup, a combination for promoting a more functional immune microenviroment could be useful. Loi et al [26], showed a correlation between reduced infiltration of TILs in residual disease in TNBC following NAC and transcriptional activity in RAS/MAPK pathways. A synergistic combination therapy with PD/PDL-1 and MEK inhibitors in mouse models of TNBC has been described [26]. Two recent trials have evaluated the efficacy of antibodies directed at PD/PDL1 in patients with TNBC with promising results [28-30]. But biomarkers for immune-checkpoints inhibitors are not yet available.

\section{Conclusions}

- Several recent clinical studies have evaluated TILs in TNBC patients with different methodological approaches. The aim of this study is to analyse the predictive value of TIL following the recommendations for TILs Evaluations in Breast Cancer by the International TILs Working Group.

- Clinicopathological biomarkers are not sufficiently accurate to distinguish the patients who have no chance of a pathologic complete response.

- In this retrospective study, we have identified different subgroups: LPBC with very high $\mathrm{pCR}$ response to NAC and non-LPBC with lacking pCR. 


\section{Conflicts of interest}

This research received a specific grant 'Beca Mutual Médica 2016’ from Fundación Mutual Médica.

The authors declared they have no competing interests.

\section{References}

1. Balko JM, Giltnane JM, and Wang K, et al (2014) Molecular profiling of the residual disease of triple-negative breast cancers after neoadjuvant chemotherapy identifies actionable therapeutic targets Cancer Discov 4(2) 223-245 https://doi.org/10.1158/21598290.CD-13-0286

2. Guarneri V, Broglio K, and Kaus SW, et al (2006) Prognostic value of pathologic complete response after primary chemotherapy in relation to hormone receptor status and other factors J Clin Oncolo 24 1037-1044 https://doi.org/10.1200/JCO.2005.02.6914

3. Hida Al, Sagara Y, and Yotsumoto D, et al (2016) Prognostic and predictive impacts of tumor-infiltrating lymphocytes differ between triple-negative and HER2-positive breast cancers treated with standard systemic therapies Breast cancer Res Treat DOI 10.1007/s10549-016-3848-2 https://doi.org/10.1007/s10549-016-3848-2 PMID: 27260189 PMCID: 4937092

4. Degnim AC, Brahmbhatt RD, Radisky DC, et al (2014) Immune cell quantitation in normal breast tissue lobules with and without lobulitis Breast cancer Res Treat 144 539-549 https://doi.org/10.1007/s10549-014-2896-8 PMID: 24596048 PMCID: $\underline{3962744}$

5. Loi S, Sirtaine N, and Piette F, et al (2013) Prognostic and predictive value of tumor-infiltrating lymphocytes in a phase III randomized adjuvant breast cancer trial in node-positive breast cancer comparing the addition of docetaxel to doxorubicin with doxorubicin-based chemotherapy: BIG 02-98 J Clin Oncol 31(7) 860-867 https://doi.org/10.1200/JC0.2011.41.0902 PMID: 23341518

6. Coates AS, Winer EP, and Goldhirsch A, et al (2015) Tailoring therapies-improving the management of early breast cancer: St Gallen International Expert Consensus on the Primary Therapy of Early Breast Cancer 2015 Ann Oncol 26(8) 1533-1546 https:// doi.org/10.1093/annonc/mdv221 PMID: 25939896 PMCID: 4 511219

7. Adams S, Gray RJ, and Demaria S, et al (2014) Prognostic value of tumor-infiltrating lymphocytes in triple-negative breast cancers from two phase III randomized adjuvant breast cancer trials: ECOG 2197 and ECOG 1199 J Clin Oncol 32(27) 2959-2966 https://doi.org/10.1200/JCO.2013.55.0491 PMID: 25071121 PMCID: 4162494

8. Dieci MV, Mathieu MC, and Guarneri V, et al (2015) Prognostic and predictive value of tumorinfiltrating lymphocytes in two phase III randomized adjuvant breast cancer trials Ann Oncol 26(8) 1698-1704 https://doi.org/10.1093/annonc/mdv239 PMID: 25995301 PMCID: $\underline{4511223}$

9. Savas P, Salgado R, and Denkert C, et al (2016) Clinical relevance of host immunity in breast cancer: from TILs to the clinic Nature 13 228-241

10. Salgado R, Denkert C, and Campbell C, et al (2015) Tumorinfiltrating lymphocytes and associations with pathological complete response and event-free survival in HER2-positive Early-stage breast cancer treated with lapatinib and trastuzumab: a secondary analysis of the NeoALTTO trial JAMA Oncol 1(4) 448-454 https://doi.org/10.1001/jamaoncol.2015.0830 PMID: 26181252

11. Denkert C, Loibl S, and Noske A, et al (2015) Tumor-associated lymphocytes as an independent predictor of response to neoadjuvant chemotherapy in breast cancer J Clin Oncol 28(1) 105-113 https://doi.org/10.1200/JC0.2009.23.7370

12. Salgado R, Denkert C, and Demaria S, et al (2015) The evaluation of tumor-infiltrating lymphocytes (TILs) in breast cancer: recommendations by an International TILs Working Group 2014 Ann Oncol 26(2) 259-271 https://doi.org/10.1093/annonc/mdu450

13. Brown M, Wittwer C (2000) Flow cytometry: principles and clinical applications in hematology Clin Chem 1221-1229 PMID: $\underline{10926916}$ 
14. Stack E, Wang C, and Roamn KA, et al (2014) Multiplexed immunohistochemistry, imaging and quantitatin:a review, with an assessment of tyramied signal amplification, multispectral imaging and mulpliplex analysis Methods $7046-78$ https://doi. org/10.1016/j.ymeth.2014.08.016 PMID: 25242720

15. Denkert C, von Minckwitz G, Brase JC, et al (2015) Tumor infiltrating lymphocytes and response to neoadjuvant chemotherapy with or without carboplatin in human epidermal growth factor receptor-2 positive and triple-negative primary breast cancers J Clin Oncol 33 983-991 https://doi.org/10.1200/JCO.2014.58.1967

16. Rooney MS, Shukla SA, and Wu CJ, et al (2015) Molecular and genetic properties of tumors associated with local immune cytolytic activity Cell 160 48-61 https://doi.org/10.1016/j.cell.2014.12.033 PMID: 25594174 PMCID: 4856474

17. Ali HR, Provenzano E, Dawson SJ, et al (2014) Association between CD8 T cell infiltration and breast cancer survival in 12439 patients Ann Oncol 25 1536-1542 https://doi.org/10.1093/annonc/mdu191 PMID: 24915873

18. Ono M, Tsuda $\mathrm{H}$, and Shimizu $\mathrm{C}$, et al (2012) Tumor-infiltrating lymphocytes are correlated with response to neoadjuvant chemotherapy in triple-negative breast cancer Breast Cancer Res Treat 132 (3) 793-805 https://doi.org/10.1007/s10549-011-1554-7

19. Demaria S, Volm MD, and Shapiro RL, et al (2007) Development of tumor-infiltrating lymphocytes in breast cancer after neoadjuvant paclitaxel chemotherapy Clin Cancer Res 7(10) 3025-3030

20. West NR, Milne K, and Truong PT, et al (2011) Tumor infiltrating lymphocytes predict response to anthracycline-based chemotherapy in estrogen receptor-negative breast cancer Breast Cancer Res 13(6) R126 https://doi.org/10.1186/bcr3072

21. Ladoire S, Arnould L, and Apetoh L, et al (2008) Pathologic complete response to neoadjuvant chemotherapy of breast carcinoma is associated with the disappearance of tumor-infiltrating foxp3+ regulatory T cells Clin Cancer Res 14(8) 2413-2420 https://doi.org/10.1158/1078-0432.CCR-07-4491 PMID: 18413832

22. Loi S, Michiels S, and Salgado R et al (2014) Tumor infiltrating lymphocytes are prognostic in triple negative breast cancer and predictive for trastuzumab benefit in early breast cancer results from the FinHER trial Ann Oncol 25 1544-1550 https://doi. org/10.1093/annonc/mdu112 PMID: 24608200

23. Simon RM, Paik S, Hayes DF (2008) Use of archived speciments in evaluation of prognostic and predictive biomarkers $\mathrm{J}$ Natl Cancer Inst 101 1446-1452 https://doi.org/10.1093/jnci/djp335

24. Cortazar P, Zhang L, and Untch M, et al (2014) Pathological complete response and long-term clinical benefit in breast cancer: the CTNeoBC pooled analysis Lancet 384 164-172 https://doi.org/10.1016/S0140-6736(13)62422-8 PMID: 24529560

25. Dieci MV, Criscitiello C, and Goubar A, et al (2014) Prognostic value of tumor-infiltrating lymphocytes on residual disease after primary chemotherapy for triple-negative breast cancer: a retrospective multicenter study Ann Oncol 25 1-8 https://doi. org/10.1093/annonc/mdt556

26. Loi S, Dushyanthen S, and Beavis PA, et al (2015) RAS/MAPK activation is associated with reduced tumor-infiltrating lymphocytes in triple-negative breast cancer therapeutic cooperation between MEK and PD-1/PD-L1 immune checkpoint inhibitors Clin Cancer Res http//dx.doi.org/10.1158/1073-0432 CCR-15-1125

27. Garcia-Martínez E, Gil GL, and Benito AC, et al (2014) Tumor -infiltrating immune cell profiles and their change after neoadjuvant chemotherapy predict response and prognosis of breast cancer Breast Cancer Res 16488 https://doi.org/10.1186/s13058014-0488-5

28. Emens. L, Braiteh FS, Cassier P, et al (2015) Inhibition of PD-L1 by MPDL3280A leads to clinical activity in patients with metastatic triple-negative breast cancer Cancer Res 75 aPD1-6 https://doi.org/10.1158/1538-7445.AM2015-2859

29. US National Library of Medicine NCT0245891.ClinicalTrials.gov. 2015 Available from: http://www.clinicaltrials.gov/ct2/show/ NCTO242589

30. Nanda R, Chow LQ, Dees EC, et al (2015) A phase lb study of pembrolizumab (MK-3475) in patients with advanced triplenegative breast cancer Cancer Res 75 aS1-09. https://doi.org/10.1158/1538-7445.SABCS14-S1-09 